\title{
Dietary microencapsulated organic acids blend enhances growth, phosphorus utilization, immune response, hepatopancreatic integrity and resistance against Vibrio harveyi in white shrimp, Litopenaeus vannamei
}

\begin{abstract}
Research on the effects of organic acid supplementations to the diets of aquacultured animals is receiving increasing global attention as a growth promoter and prophylactic against bacterial pathogens. Three separate experiments were conducted on Pacific white shrimp, Litopenaeus vannamei, when fed diets supplemented with different levels of a microencapsulated organic acids blend $(\mathrm{OAB})$ at $0 \%$ (control), 1\%, 2\%, or $4 \%$. The first experiment was performed to examine the growth performance, phenoloxidase (PO) activity, total presumptive Vibrio spp. colony forming units (CFU) in the hepatopancreas and histology of the hepatopancreas of the post larvae (PL 36) shrimp (initial weight $0.123 \pm$ $0.010 \mathrm{~g}$ ) fed these diets after 50 days. Results showed shrimp fed the $2 \%$ OAB diet had significantly higher $(\mathrm{P}<0.05)$ growth, lower $(\mathrm{P}=0.132)$ hepatopancreatic Vibrio spp. $\mathrm{CFU}$ counts with substantially more lipid storage cells in the hepatopancreas than those fed the control diet. No significant effect on PO activity was detected $(\mathrm{P}>0.05)$. In the second experiment, dry matter and phosphorus digestibility was measured and results showed the $2 \%$ OAB diet led to significantly better digestibility than the control treatment. For the third experiment, the resistance of the shrimp to Vibrio harveyi was investigated over 10 days and subsequent effects to PO activity and hepatopancreatic histopathology determined. Results demonstrated that the $1 \%$ and $2 \% \mathrm{OAB}$ diets led to significantly higher survival than those fed the control or $4 \%$ OAB diet. PO activity was significantly higher for shrimp fed the OAB supplemented diets as well showing less hepatopancreatic damage. These findings indicate that the $\mathrm{OAB}$ used can substantially improve productivity and resistance to pathogenic bacteria and may be a viable alternative to the use of antibiotics in the shrimp industry. This is the first study showing the hepatopancreatic protective properties of dietary organic acids against vibriosis in shrimp.
\end{abstract}

Keyword: Organic acids; Antimicrobials; White shrimp; Vibriosis; Hepatopancreatic histology 